\title{
1 Blue-shifted deep ocean currents in the equatorial
}

\section{Indian Ocean}

\author{
3 P. Amol ${ }^{* 1}$. Vineet Jain ${ }^{2}$. S. G. Aparna ${ }^{2}$ \\ 5 the date of receipt and acceptance should be inserted later
}

6 Abstract Spectra from two decades of zonal current data at $\sim 4000 \mathrm{~m}$ in the

7 central and western equatorial Indian Ocean show a shift in the dominant fre-

8 quencies from the west to the east. The $120-180$-day period is stronger at $77^{\circ} \mathrm{E}$,

9 the $60-120$-day period at $83^{\circ} \mathrm{E}$, and the $30-90$-day period at $93^{\circ} \mathrm{E}$. The weakening

10 of lower frequencies near the eastern boundary can be explained using theoretical

11 ray paths of Kelvin waves and reflected Rossby waves. The equatorial Kelvin wave

12 forced by winds reflects from the eastern boundary as Rossby waves with different

13 meridional modes. After reflection, the low (high) frequency Rossby beams travel

14 a larger (shorter) distance before reaching the bottom, thereby creating a shadow

15 zone, a region with low wave energy, between the ray path and the eastern bound-

16 ary. The shift in frequency with longitude is not evident in the top $1000 \mathrm{~m}$, where

17 the current is dominated by the semi-annual cycle.

\footnotetext{
* Corresponding author

${ }^{1}$ CSIR-National Institute of Oceanography, Regional Centre, Visakhapatnam, India

${ }^{2}$ CSIR-National Institute of Oceanography, Dona Paula, Goa, India
} 
${ }_{18}$ Keywords Equatorial Ocean · Bottom currents · Indian Ocean · Rossby waves ·

19 Kelvin waves · Ray path

\section{$20 \quad 1$ Introduction}

Observations from the equatorial Indian Ocean show energetic current fluctuations

22 in the deeper waters (Luyten and Swallow 1976; Eriksen 1980; O’Neill and Luyten 1984; Ponte and Luyten 1990; Dengler and Quadfasel 2002). The zonal currents throughout the water column are characterised by complex vertical structures with time scales longer than a month and are trapped within few degrees of the equator. Several attempts were made to explain the energy available for the sustenance of such deep ocean currents, and one of the possible reasons is the direct transmission of wind-forced energy into deeper waters.

Numerical solutions have shown that periodic zonal winds generate a welldefined Kelvin beam that propagates both eastwards and downwards (McCreary 1984; Rothstein et al. 1985). The Kelvin beam reflects from the eastern boundary

32 as a Rossby beam, which has a steeper ray path that can reach the bottom of the ocean at seasonal time scales. The signatures of these beams are evident in the observations from the equatorial Indian Ocean. Luyten and Roemmich (1982) showed that the mid-depth zonal currents in the western equatorial Indian ocean were dominated by the semi-annual cycle. They suggested that these fluctuations were caused by equatorially trapped Kelvin waves and first meridional mode Rossby waves. Huang et al. (2018) arrived at the same conclusion in the central and eastern equatorial Indian Ocean using an oceanic reanalysis product. They showed

${ }_{40}$ the enhancement of the semi-annual cycle and the associated phase propagation 
41 in the intermediate depths $(200-1500 \mathrm{~m})$ of the central equatorial Indian Ocean

42 were caused by reflected first-mode Rossby waves.

The transmission of wind-forced energy into the deeper waters will, however,

44 be affected by the presence of a strong pycnocline (Philander 1978). Gent and

45 Luyten (1985) showed that there was considerable reflection of energy from the

${ }_{46}$ pycnocline, which prevented the transmission of wind-forced energy into deeper

47 waters. Their study only described a single reflection, and a complete picture

48 involves re-reflections of energy from the surface (Kessler and McCreary 1993).

49 With each reflection, a small fraction of energy would leak into the deep ocean.

50 In addition, Rothstein et al. (1985) suggested that the vertical mixing could cause

51 model solutions to lose their beam-like characteristics, which is probably why the

52 simulations, like that by Philander and Pacanowski (1981), show strong trapping

53 of energy near the surface. Some studies have also shown that random fluctuations

${ }_{54}$ in density could cause waves to decay in the direction of energy propagation more

55 rapidly (Tang and Mysak 1976; Mysak 1978). If the beam weakens with depth

56 owing to damping and variations in density, can the wind energy flux reach the

${ }_{57}$ bottom of the ocean and impact the deep ocean circulation?

58 In this study, we use spectra of deep zonal currents $(\sim 4000 \mathrm{~m})$ obtained from

59 the data collected during 2000-2020 to show signatures of wind-driven circulation

${ }_{60}$ in the near-bottom currents. The dominant frequencies blue shift with longitude

61 - the $120-180$-day period is stronger at $77^{\circ} \mathrm{E}$, the $60-120$-day period at $83^{\circ} \mathrm{E}$,

62 and the $30-90$-day period at $93^{\circ} \mathrm{E}$ - and this shift in frequency can be explained

63 using theoretical ray paths of Kelvin and Rossby waves. The signatures of these

64 waves propagating downwards as equatorial beams are also evident in the current

65 observations from the near-surface and intermediate depths. 
The rest of the paper is organised as follows. Sec. 2 describes the data used in this study. The spectra of the $\sim 4000 \mathrm{~m}$ zonal currents are shown in Sec. 3. The effect of ray paths and shadow zones in causing a shift towards higher frequencies in the eastern Indian Ocean is detailed out in Sec. 4. Sec. 5 summarises and concludes the study.

\section{Data and Methods}

We used data from the deep-sea equatorial mooring programme, which is under Ocean Observation Network (OON) programme of ESSO-Indian National Centre for Ocean Information Services (INCOIS), India (Jain et al. 2021). The currents were measured at three locations: $77^{\circ} \mathrm{E}, 83^{\circ} \mathrm{E}$, and $93^{\circ} \mathrm{E}$ (Fig. 1a). The data from the top 1400 m were obtained from Acoustic Doppler Current Profilers (ADCP), and the vertical and temporal resolution was $16 \mathrm{~m}$ and one hour, respectively. Recording current meters (RCMs) with the same temporal resolution were used to measure currents at $\sim 1000, \sim 2000 \mathrm{~m}$ and $\sim 4000 \mathrm{~m}$ depths. The depth of the water column at these three locations vary between 4400 and $4700 \mathrm{~m}$. The data were collected from 2000-2020 and were discontinuous, with gaps ranging from few days to few years. The data at $93^{\circ} \mathrm{E}$ were available only till 2014 . The details of mooring configuration and data availability are available in Jain et al. (2021).

As there were extensive gaps in the ADCP data, we used the Lomb-Scargle periodogram to compute the spectra (Lomb 1976; Scargle 1982). Unlike the fast Fourier transform, this approach computes the power spectral density of unevenly spaced data sets or, in this case, data sets with gaps. The periodogram was normalised using residuals of data around the constant reference model. The signifi- 
cance test or the false alarm probability was computed using the bootstrap method

(see VanderPlas (2018) for details related to Lomb-Scargle periodogram and false

alarm probability).

The wind data from 2000-2016 were obtained by merging QuikSCAT and AS-

93 CAT satellite data products (https://coastwatch.pfeg.noaa.gov/). The tem-

94 perature and salinity data for computing Brunt-Väisälä frequency profile were

95 from the World Ocean Atlas (Boyer et al. 2018).

\section{Spectra of the bottom currents}

In this section, we show basic statistics and spectra for the near-bottom zonal currents at $\sim 4000 \mathrm{~m}$. The magnitude of currents at $\sim 4000 \mathrm{~m}$ ranges from -12 to 12 $\mathrm{cm} \mathrm{s}^{-1}$ and the mean flow is close to zero at all locations (Fig. 1b-d). The standard deviation is $4.2 \mathrm{~cm} \mathrm{~s}^{-1}$ at $77^{\circ} \mathrm{E}$ and drops to $3.2 \mathrm{~cm} \mathrm{~s}^{-1}$ at $93^{\circ} \mathrm{E}$. In comparison, the maximum magnitude is greater than $100 \mathrm{~cm} \mathrm{~s}^{-1}$ for near-surface currents and decreases to $30-50 \mathrm{~cm} \mathrm{~s}^{-1}$ at intermediate depths of 200-1000 m (Jain et al. 2021). Similarly, the mean flow is eastward $\left(15-60 \mathrm{~cm} \mathrm{~s}^{-1}\right)$ for the near-surface current and close to zero for the intermediate current. The near-bottom currents at all three locations are de-correlated.

The Lomb-periodogram for the bottom currents shows that the dominant frequencies blue-shifts with longitude (Fig. 2a-c). The lower frequencies are stronger at the western mooring $\left(77^{\circ} \mathrm{E}\right)$, while the higher frequencies are stronger at the eastern mooring $\left(93^{\circ} \mathrm{E}\right)$. At $77^{\circ} \mathrm{E}$, the seasonal cycles, like the 120 -day, 160-day and 180-day period, dominate (Fig. 2a). The 90-day and 60-day peaks are also evident, but they are weaker compared to the seasonal cycles. At $83^{\circ} \mathrm{E}$, the $60-$ 
The spectra also shows a frequency shift with depth at $93^{\circ} \mathrm{E}$ (Fig. 2f). The $30-$ 50-day peak, which is the dominant band at $4000 \mathrm{~m}$, weakens at $2000 \mathrm{~m}$. At this depth, the 90-day peak is the strongest. The intraseasonal periods further weakens at $1000 \mathrm{~m}$ and the seasonal cycles, like 120-day, become stronger. Between 150-500 $\mathrm{m}$, the semi-annual cycle is the strongest.

The surface circulation in the equatorial Indian Ocean is known for its strong semi-annual cycle. The spectra shows that the cycle is the strongest between $500-$ $1500 \mathrm{~m}(200-500 \mathrm{~m})$ depth at $77^{\circ} \mathrm{E}$ and $83^{\circ} \mathrm{E}\left(93^{\circ} \mathrm{E}\right.$, Fig. 2d-i). Huang et al. (2018) also found a maximum variance for the semi-annual harmonic in this depth range. Though the semi-annual cycle is strong near the surface at all locations (Fig. 2d,e), the amplitude of the higher frequencies becomes comparable at $93^{\circ} \mathrm{E}$ (Fig. 2f). The relatively weak semi-annual period and strong intraseasonal periods at $93^{\circ} \mathrm{E}$ at intermediate depths were reported by Chen et al. (2020) using ADCP data from $2015-2019$. 


\section{Equatorial beams}

In this section, we use the ray paths derived from 'equatorial beam theory' (McCreary 1984) along with observations from the equatorial moorings to explain the shift in dominant frequencies observed in the bottom currents. We first show how the ray paths and shadow zones (or low wave energy zones) of large-scale waves vary based on the property of the wave and the medium (Section 4.1). We then compare the theoretical ray paths with observed spectra and highlight the role of beams in causing a shift in dominant frequencies along the longitude (Section 4.2).

\subsection{The shifting shadow zones}

We define shadow zones as regions in the equatorial ocean where large-scale waves are not detected due to the presence of continental boundaries or topographical obstructions, like ridges and islands. The area of the shadow zone depends on the frequency of waves (Fig. 3d), stratification (Fig. 3e), the width of the basin (Fig. 3f), and in the case of Rossby waves, the meridional mode number (Fig. 3c).

Based on Wentzel-Kramers-Brillouin (WKB) approximation, the slope of the ray path is given by $\omega / N_{b}(z)$ for Kelvin waves and $(2 n+1) \omega / N_{b}(z)$ for Rossby waves, where $\omega$ is the frequency and $N_{b}(z)$ is the background Brunt-Väisälä frequency profile. The climatological $N_{b}(z)$ profiles are averaged from $45^{\circ} \mathrm{E}-98^{\circ} \mathrm{E}$ along the equator. The details regarding the approximations for computing the ray path can be found in McCreary (1984).

Fig. 3a shows a schematic of beams generated by 150-210-day band winds at $77^{\circ} \mathrm{E}$. The $N_{b}(z)$ used to calculate the ray path is the annual climatology. The beam travelling towards the west from the wind source is the incident Rossby wave (only 
the first meridional mode shown), and the beam travelling towards the east is the equatorial Kelvin wave. The Kelvin wave also reflects from the eastern boundary as a first meridional mode Rossby wave. As the wave propagates downwards along its ray path, its energy would drop and spread owing to wave damping or vertical mixing. Numerical simulations by McCreary (1984) show that the reflected Rossby waves from an eastern boundary can cause more energy to propagate into the deep ocean compared to the directly forced waves generated in an unbounded solution.

If the wind blows all along the equator, similar ray paths for each wave can be plotted at all longitudes. For a given basin size, the region occupied by all these ray paths (Fig. 3b) will be constrained by the ray paths of the Kelvin wave and the incident Rossby wave generated near the western and eastern boundaries, respectively (solid lines in Fig. 3b). For example, the Kelvin wave will be trapped within the top $250 \mathrm{~m}$ of the ocean, and the width of this trapping increases from west to east based on the slope of the ray path. In this region, the Kelvin wave does not occur in isolation and can superpose with either the incident or the reflected Rossby waves. The ray paths of the incident Rossby wave cover a major fraction of the ocean basin (almost the entire water column in the western Indian Ocean), whereas the ray paths of the first mode reflected Rossby waves is confined to the eastern and central Indian Ocean. The grey shaded region is the shadow zone, which forms below the ray path of the reflected Rossby waves.

Higher meridional modes (Fig. 3c) and higher frequencies (Fig. 3d) have steeper ray paths and smaller shadow zones. Kelvin wave is a single mode wave; hence, after reflection, only the width of the shadow zone varies with each meridional mode of the Rossby wave (Fig. 3c). Based on the aforementioned ray path equation, the shadow zone of the Rossby wave would disappear if the number of meridional 
modes tends to infinity. However, the equation is an approximation and holds only for low-frequency, low-wavenumber, inviscid Rossby waves. At higher meridional modes, there are fewer baroclinic modes that can strongly couple with winds at seasonal and intraseasonal time scales, and it would be unreasonable to associate the higher meridional modes with vertical propagation of energy as several baroclinic modes must superpose to form beams (McCreary 1984; Mukherjee et al. 2018).

If the frequency increases, both the width and the height of the shadow zone would decrease (Fig. 3d). The decrease in the width of the shadow zone with frequency explains the observed shift in spectral peaks in the bottom currents (see Section 4.2 for more details). A similar change in the height of the shadow zone would cause a shift in the frequency with depth; the lower (higher) frequencies will be stronger near the surface (bottom). This shift is evident at $93^{\circ} \mathrm{E}$ mooring, even though the vertical resolution of the data is very coarse below $500 \mathrm{~m}$ (Fig. 2i).

The area of the shadow zone also depends on the size of the basin; as the basin size increases, the width and the height of the shadow zone decreases. For a given basin size, there is a cut-off period below which the shadow zone will not form. For $4000 \mathrm{~m}$ depth, the cut-off period is $\sim 135$ days for the Pacific Ocean and $\sim 50$ days for the Indian Ocean and the Atlantic Ocean. Fig. 3f shows that there is no shadow zone for the 90-day period in the Pacific Ocean, whose basin width is much larger than that of the Indian Ocean. At lower frequencies, like the annual cycle, the beam slope is so small that it takes more than $\sim 25,000 \mathrm{~km}$ for the beam to reach $1000 \mathrm{~m}$ depth (Rothstein et al. 1985).

In general, we can ignore the effect of stratification as they cause only small changes in the ray path (Fig. 3e). Rossby waves can also reflect from the western 
boundary or the bottom of the ocean and can create complex structures (McCreary 1984). In the case of bottom reflection, the phase (energy) would propagate downwards (upwards).

\subsection{Observational evidence for beams}

The observed frequency shift at $\sim 4000 \mathrm{~m}$ can be explained using the shadow zones of the reflected Rossby waves from the eastern boundary. Fig. 4 shows ray paths for both Kelvin and Rossby waves for four periods: 180, 120, 90, and 30 days. The shadow zone is marked as hatched lines for the fifth meridional mode. The ray paths are plotted from the eastern and western boundaries as they represent the extreme case for the directly forced and reflected waves (similar to Fig. 3b). In reality, the beam path changes based on the location of the wind forcing, which varies along the equator (Fig. 5). Strong spectral peaks within the vicinity of the incident or eastern boundary Rossby wave path (orange and solid red lines in Fig. 4) suggest that the wind forcing is strong in the eastern Indian Ocean. Similarly, strong peaks near the reflected Rossby wave path (green and purple dashed line) traced using a western boundary Kelvin wave (solid blue line) suggest that the winds are strong in the western Indian Ocean. Note that, unless specifically mentioned, we discuss only the Kelvin and first mode Rossby ray paths here, and the fifth mode Rossby ray paths are shown only for reference.

The 180-day ray path in Fig. 4a follows the result obtained by Huang et al. (2018). The beam path of the first-mode reflected Rossby and Kelvin waves coincides with enhanced spectral power in the upper $1500 \mathrm{~m}$ (Fig. 4). Huang et al. (2018) showed that the variance explained in this depth range extended only till 
$65^{\circ} \mathrm{E}$, west of which the variance is weaker. The significant power observed below $1500 \mathrm{~m}$ could be due to the higher-order meridional modes or vertical mixing, which could weaken and broaden the beam both vertically and meridionally (McCreary 1984).

The wind spectra show that the semi-annual cycle is strong along the entire basin, and the maximum amplitude is observed between $60^{\circ} \mathrm{E}-80^{\circ} \mathrm{E}$ (Fig. 5). Therefore, the peak would be expected somewhere between the reflected Rossby (dashed curve) and the incident Rossby wave path. The maximum amplitude for the 180 -day signal is observed at $\sim 680 \mathrm{~m}$ at $77^{\circ} \mathrm{E}$ and $200 \mathrm{~m}$ at $93^{\circ} \mathrm{E}$.

The 120-day ray path is similar to the semi-annual cycle (Fig. 4b). The spectral power enhances along the Kelvin and first-mode reflected Rossby ray paths. As both Kelvin and Rossby ray paths cross each other between $200-600 \mathrm{~m}$ at $83^{\circ} \mathrm{E}$, it is difficult to separate them at this depth range. At $77^{\circ} \mathrm{E}$, the maximum amplitude is observed at $2000 \mathrm{~m}$ depth and is in the vicinity of the first-mode reflected Rossby wave path. The 120-day wind spectra show a bimodal structure with power weakening in the central Indian Ocean (Fig. 5). This weakening of power is also evident between the reflected and incident Rossby ray paths (Fig. 4b).

The 90-day power enhances along Rossby ray paths generated by winds near the eastern (solid line) and the western (dashed line) Indian Ocean (Fig. 4c). The strongest power is observed at $1000 \mathrm{~m}$ at $77^{\circ} \mathrm{E}$, which lies within the vicinity of the incident Rossby ray path. Like the 120 days, the winds in 90-100-day band also have a bimodal structure: the $\sim 95$ day is strong in the eastern Indian Ocean and $\sim 90$ day is strong in the western Indian Ocean (Fig. 5). Han (2005) showed that even though the winds are relatively weak, the Indian Ocean selectively responds to the 90-day forcing, owing to the resonance excitation of second-baroclinic mode 
waves. The resonance is more concentrated towards the eastern Indian Ocean because the westward propagating Rossby wave is slower compared to the eastward propagating Kelvin wave (Han et al. 2011). Nevertheless, the 90-day period peaks at $4000 \mathrm{~m}(2000 \mathrm{~m})$ at $83^{\circ} \mathrm{E}\left(77^{\circ} \mathrm{E}\right)$ along the reflected Rossby ray path, which suggests that the winds in the western Indian Ocean are also important in driving the currents at deeper depths.

The 30-day period is the minimum period of the Rossby wave, and there is no shadow zone for this period (Fig. 4d). The minimum period is an approximation and would vary based on the speed of the first baroclinic mode Kelvin wave. For $c=3 \mathrm{~ms}^{-1}$, the minimum period is 30.4 days. The beam generation for Rossby waves at higher frequencies, in general, is unlikely, because these waves are dispersive and cannot retain their coherency as wave packets. The non-dispersive Kelvin waves, however, can reach the bottom of the ocean and enhance the power east of $80^{\circ} \mathrm{E}$. The presence of the Central Indian Ridge at $75^{\circ} \mathrm{E}$ creates a local shadow zone for high-frequency Kelvin waves generated by winds in the western Indian Ocean. The spectra show that the winds in the 30-60-day band are stronger between $60-90^{\circ} \mathrm{E}$ (Fig. 5), and it is the energy from the wind forcing at $\sim 60^{\circ} \mathrm{E}$ that reaches the bottom at $93^{\circ} \mathrm{E}$ (see thin blue line in Fig. 4d). Though beams generated between $55-58^{\circ} \mathrm{E}$ can also reach the bottom, they will be obstructed by the Ninety East Ridge.

In summary, the energy from the spectra is the weakest in the shadow zones of the reflected Rossby waves, and the blue shift in dominant frequencies of the bottom currents is linked to the shift in the width of the shadow zone (Fig. 3d). The dominant frequency also changes with depth because of the change in the height of the shadow zone and is evident at $93^{\circ} \mathrm{E}$ (Fig. 2i). 
5 Discussion

The surface circulation in the equatorial Indian Ocean is dominated by the semiannual cycle. The eastward jets that occur during the boreal spring and autumn are known as the Wyrtki Jets (Wyrtki 1973). These jets are known to be driven by zonal winds with current speeds that can peak more than $2 \mathrm{~m} \mathrm{~s}^{-1}$. The impact of these winds are stronger in the top 200-300 m below which strong upward phase propagation is evident (Jain et al. 2021; McPhaden et al. 2015). Our study shows that the dominance of the semi-annual cycle does not extend till the bottom of the ocean, particularly in the eastern Indian Ocean. Near the bottom, the lower frequencies are stronger at $77^{\circ} \mathrm{E}$ and become weaker towards the east. The shift in frequencies is caused by the vertical propagation of energy generated by surface winds. The wind-forced Kelvin waves reflect from the eastern boundary as Rossby waves and propagate downwards. The ray path of Rossby waves has a larger slope compared to Kelvin wave and can reach the bottom of the ocean even at seasonal time scales. At higher frequencies, there are no Rossby waves, but the ray path of the Kelvin waves is steeper and can reach the bottom of the ocean.

Based on the linear-wave theory, one may expect weak spectral power in the shadow zones and anything outside would be a complex superposition of several modes generated by winds all along the equatorial region. The spectral analysis shows that the strong spectral power roughly coincides with the beam path of the first-mode reflected Rossby waves (Fig. 4). This result is consistent with that shown in earlier studies (Luyten and Roemmich 1982; Huang et al. 2018; Zanowski and Johnson 2019). The observed amplification of wave energy along the first-mode reflected Rossby ray path could be due to the following reasons. 
For vertical propagation of energy, several baroclinic modes of a meridional mode should superpose, but higher meridional modes have only fewer baroclinic modes that can strongly couple with winds and hence are weaker. The effect of the even meridional modes would be negligible because the Hermite polynomials are antisymmetric and have a zero-crossing at the equator. In addition, higher modes have shorter wavelengths and can be diffused more easily by friction.

The spectral analysis shows that there is significant energy evident in the shadow zone of the first mode ray path. For example, the 180-day peak is one of the dominant peaks for the bottom currents at $93^{\circ} \mathrm{E}$ (Fig. 2c). It is difficult to attribute the energy observed in the shadow zone to higher meridional modes because it is possible that the wave energy could spread out along the ray path due to vertical mixing (McCreary 1984; Rothstein et al. 1985). Also, the 180day period is the strongest period in the equatorial Indian Ocean, and the energy would penetrate deeper compared to that from other periods before it is completely dissipated.

The analysis presented here is not without caveats. The ray path is calculated based on the WKB approximation, which is not valid in the upper few hundred meters because of the sharp changes in the density profile. For the beam to penetrate the thermocline, the vertical scale of the oscillations should be much smaller than the background variations of $N_{b}^{2}$. A typical vertical wavelength for the first mode is $4900 \mathrm{~m}$ (60 cm equivalent depth), and this wavelength is almost seven times more than the wavelength of waves that can freely pass through the thermocline (Moore and Philander 1977; Boyd 2018). In addition, Gent and Luyten (1985) showed that for an equivalent depth between $1 \mathrm{~cm}$ and $1 \mathrm{~m}$ only $10-20 \%$ of the energy could pass through the thermocline. As the ocean surface acts like 
a rigid lid, one must also consider the effects of re-reflection; with each reflection, more fraction of energy would penetrate the thermocline. In addition, the wind-forcing extends deep into the mixed layer, and its impact on energy transfer through turbulent processes is not well understood.

The downward propagation of energy can also be modified by the presence of mid-oceanic ridges and islands. They can create a local shadow zone, cause re-reflections, and affect the resonance amplitude of the baroclinic modes. For example, Chen et al. (2020) showed that Maldives islands could cause abrupt changes in the amplitude of the equatorial intermediate current. However, as the islands lie on the nodes of the first four baroclinic modes, the resonance amplitude is not affected.

In spite of the above-mentioned limitations, the spectra of the zonal currents show an enhancement of energy along the Kelvin and Rossby ray paths, suggesting that the surface forcing from the winds reaches the bottom of the ocean. The transmission of energy is evident for both seasonal and intraseasonal time scales, which explains the observed frequency shift near the bottom of the ocean.

Deep ocean currents are known to be driven by differences in water density. They play an important role in the transport of water masses across the ocean basin and significantly impact climate scale processes. Earlier studies have shown signatures of Madden-Julian Oscillations in the deeper oceans (Matthews et al. 2007; Webber et al. 2014). Unlike the surface circulation, the spatial distribution of the intraseasonal frequencies and climate modes, such as El Niño and Indian Ocean Dipole, near the bottom would be determined by the ray path of these 351 large-scale waves. The downward propagation of energy by surface winds would 52 also play an important role in the deep ocean biogeochemistry. 
Acknowledgements The authors thank Julian P. McCreary for sharing his insights on the equatorial beam theory and D. Shankar for his critical comments. The support from the CSIRNIO mooring group (V. Fernando, N P Satelkar, S. T. Khalap, S. Ghatkar, P. A. Tari, and M. G. Gaonkar), ship cell members (M S Kerkar, S P Vernekar and A C D'Souza), officers, crew, and seamen from various cruises is gratefully acknowledged. The mooring operations were carried out on the research vessels O R V Sagar Kanya, R V Sindhu Sadhana, and T D V Sagar Nidhi. The authors also thank V. S. N. Murty and his colleagues for overseeing the deep-sea mooring programme from 2000-2017. This is CSIR-NIO contribution ????.

\section{Declarations}

Funding

Financial support for this project was provided by Ministry of Earth Sciences

(MoES), India via their Ocean Observation Network (OON) programme and by Council of Scientific and Industrial Research (CSIR).

Conflict of interest

The authors declare that they have no conflict of interest.

Data availability

The ocean current data are available from https://incois.gov.in/essdp/. The wind data were obtained https://coastwatch.pfeg.noaa.gov/. The temperature and salinity data are from https://www.ncei.noaa.gov/products/world-ocean-atlas. 
Code availability

Python packages (astropy, numpy, scipy, matplotlib, and netCDF4) were used for analysis and graphics.

Authors' contributions

- P. Amol: Conceived and designed the study; acquired, analysed, and interpreted the data; drafted the article.

- V. Jain: Acquired and analysed the data; drafted the article; implemented computer programs.

- S. G. Aparna: Processed and organised the data; reviewed and edited the article.

\section{References}

Boyd JP (2018) Dynamics of the equatorial ocean. Springer-Verlag Berlin Heidelberg, https: //doi.org/10.1007/978-3-662-55476-0

Boyer TP, Garcia HE, Locarnini RA, Zweng MM, Mishonov AV, Reagan JR, Weathers KA, Baranova OK, Paver CR, Seidov D, Smolyar IV (2018) World Ocean Atlas 2018. NOAA National Centers for Environmental Information https://www.ncei.noaa.gov/archive/ accession/NCEI-WOA18

Chen G, Han W, Zhang X, Liang L, Xue H, Huang K, He Y, Li J, Wang D (2020) Determination of Spatiotemporal Variability of the Indian Equatorial Intermediate Current. Journal of Physical Oceanography 50(11):3095-3108, https://doi.org/10.1175/JP0-D-20-0042.1 
Dengler M, Quadfasel D (2002) Equatorial deep jets and abyssal mixing in the Indian Ocean. Journal of Physical Oceanography 32(4):1165-1180, https://doi.org/10.1175/ $1520-0485(2002) 032<1165:$ EDJAAM $>2.0 . \mathrm{CO} ; 2$

Eriksen CC (1980) Evidence for a continuous spectrum of equatorial waves in the Indian Ocean. Journal of Geophysical Research: Oceans 85(C6):3285-3303, https://doi.org/10.1029/ JC085iC06p03285

Gent PR, Luyten JR (1985) How much energy propagates vertically in the equatorial oceans? Journal of Physical Oceanography 15(7):997-1007, https://doi.org/10.1175/ $1520-0485(1985) 015<0997:$ HMEPVI $>2.0 . \mathrm{CO} ; 2$

Han W (2005) Origins and dynamics of the 90-day and 30-60-day variations in the equatorial Indian Ocean. Journal of Physical Oceanography 35(5):708-728, https://doi.org/10. $1175 / J P 02725.1$

Han W, McCreary JP, Masumoto Y, Vialard J, Duncan B (2011) Basin resonances in the equatorial Indian Ocean. Journal of Physical Oceanography 41(6):1252-1270, https:// doi.org/10.1175/2011JP04591.1

Huang K, McPhaden MJ, Wang D, Wang W, Xie Q, Chen J, Shu Y, Wang Q, Li J, Yao J (2018) Vertical propagation of middepth zonal currents associated with surface wind forcing in the equatorial Indian Ocean. Journal of Geophysical Research: Oceans 123(10):7290-7307, https://doi.org/10.1029/2018JC013977

Jain V, Amol P, Aparna S, Fernando V, Kankonkar A, Murty V, Almeida A, Sardar AA, Khalap S, Satelkar N, et al. (2021) Two decades of current observations in the equatorial Indian Ocean. Journal of Earth System Science 130(2):1-8, https://doi.org/10.1007/ s12040-021-01568-4

Kessler WS, McCreary JP (1993) The annual wind-driven Rossby wave in the subthermocline equatorial Pacific. Journal of Physical Oceanography 23(6):1192-1207, https://doi .org/ 10.1175/1520-0485(1993) 023<1192: TAWDRW>2.0.CO;2

Lomb NR (1976) Least-squares frequency analysis of unequally spaced data. Astrophysics and space science 39(2):447-462, https ://doi.org/10.1007/BF00648343

Luyten JR, Roemmich DH (1982) Equatorial currents at semi-annual period in the Indian Ocean. Journal of Physical Oceanography 12(5):406-413, https://doi.org/10.1175/ 
$1520-0485(1982) 012<0406:$ ECASAP $>2.0 . C 0 ; 2$

Luyten JR, Swallow J (1976) Equatorial undercurrents. Deep Sea Research and Oceanographic Abstracts 23(10):999-1001, https://doi.org/10.1016/0011-7471(76)90830-5 Matthews AJ, Singhruck P, Heywood KJ (2007) Deep ocean impact of a Madden-Julian Oscillation observed by Argo floats. Science 318(5857):1765-1769, https://doi.org/10.1126/ science. 1147312

McCreary JP (1984) Equatorial beams. Journal of Marine Research 42(2):395-430, https: //doi.org/10.1357/002224084788502792

McPhaden MJ, Wang Y, Ravichandran M (2015) Volume transports of the W yrtki jets and their relationship to the Indian Ocean Dipole. Journal of Geophysical Research: Oceans 120(8):5302-5317, https://doi.org/10.1002/2015JC010901

Moore DW, Philander SGH (1977) Modelling of the tropical ocean circulation, The Sea, vol 6. John Wiley \& Sons, New York

Mukherjee A, Shankar D, Chatterjee A, Vinayachandran P (2018) Numerical simulation of the observed near-surface east india coastal current on the continental slope. Climate Dynamics 50(11):3949-3980, https://doi.org/10.1007/s00382-017-3856-x

Mysak LA (1978) Wave propagation in random media, with oceanic applications. Reviews of Geophysics 16(2):233-261, https://doi.org/10.1029/RG016i002p00233

O'Neill K, Luyten JR (1984) Equatorial velocity profiles. Part II: Zonal component. Journal of Physical Oceanography 14(12):1842-1852, https ://doi.org/10.1175/1520-0485(1984) $014<1842:$ EVPPIZ>2.0. CO 2

Philander S (1978) Forced oceanic waves. Reviews of Geophysics 16(1):15-46, https://doi. org/10.1029/RG016i001p00015

Philander S, Pacanowski R (1981) Response of equatorial oceans to periodic forcing. Journal of Geophysical Research: Oceans 86(C3):1903-1916, https://doi.org/10.1029/ JC086iC03p01903

Ponte RM, Luyten J (1990) Deep velocity measurements in the western equatorial Indian Ocean. Journal of Physical Oceanography 20(1):44-52, https://doi.org/10.1175/ $1520-0485$ (1990) 020<0044: DVMITW>2.0. CO ; 2 
Rothstein LM, Moore DW, McCreary JP (1985) Interior reflections of a periodically forced equatorial Kelvin wave. Journal of Physical Oceanography 15(7):985-996, https://doi. $\operatorname{org} / 10.1175 / 1520-0485(1985) 015<0985$ : IROAPF > 2.0.CO;2

Scargle JD (1982) Studies in astronomical time series analysis. ii-statistical aspects of spectral analysis of unevenly spaced data. The Astrophysical Journal 263:835-853, https://doi. $\operatorname{org} / 10.1086 / 160554$

Sindhu B, Suresh I, Unnikrishnan A, Bhatkar N, Neetu S, Michael G (2007) Improved bathymetric datasets for the shallow water regions in the Indian Ocean. Journal of Earth System Science 116(3):261-274, https://doi.org/10.1007/s12040-007-0025-3

Tang C, Mysak L (1976) A note on "Internal waves in a randomly stratified fluid". Journal of Physical Oceanography 6(2):243-246, https://doi.org/10.1175/1520-0485(1976) 006<0243: ANOWIA>2.0.CO;2

VanderPlas JT (2018) Understanding the Lomb-Scargle periodogram. The Astrophysical Journal Supplement Series 236(1):16

Webber BG, Matthews AJ, Heywood KJ, Kaiser J, Schmidtko S (2014) Seaglider observations of equatorial Indian Ocean Rossby waves associated with the Madden-Julian Oscillation. Journal of Geophysical Research: Oceans 119(6):3714-3731, https://doi.org/10.1002/ 2013JC009657

Wyrtki K (1973) An equatorial jet in the indian ocean. Science 181(4096):262-264, 10.1126/ science.181.4096.262

Zanowski H, Johnson GC (2019) Semiannual variations in 1,000-dbar equatorial indian ocean velocity and isotherm displacements from argo data. Journal of Geophysical Research: Oceans 124(12):9507-9516, https://doi.org/10.1029/2019JC015342 
Fig. 1: (a) Map showing the mooring locations. The background is the bathymetry from Sindhu et al. (2007). The inset shows map of north Indian Ocean. The red dashed line is the region of interest. Zonal currents at $4000 \mathrm{~m}$ at (b) $77^{\circ} \mathrm{E}$, (c) $83^{\circ} \mathrm{E}$, and $(\mathrm{d}) 93^{\circ} \mathrm{E}$.

Fig. 2 Normalized Lomb periodogram of zonal currents at $4000 \mathrm{~m}$ depth: (a) $77^{\circ} \mathrm{E}$, (b) $83^{\circ} \mathrm{E}$, and (c) $93^{\circ} \mathrm{E}$. The dashed horizontal line shows the $1 \%$ false alarm probability. The gray shaded region highlights the shift in dominant frequencies from west to east. (d)-(f) Same as (a)-(c) but for currents at 60 m (black) and 150$500 \mathrm{~m}$ (red). The spectra for the 150-500 m current is computed after averaging the current over this depth range. Note that the ordinate does not have the same limits. (g)-(i) Normalized Lomb periodogram of zonal currents with depth. As the difference in power is large between the surface and bottom currents, the periodogram is smoothed and plotted in log scale to highlight the shift in dominant frequency with depth. The hatched area is equivalent to the gray shaded region in panels (a)-(c).

Fig. 3 Schematic of the ray paths in the equatorial Indian Ocean. (a) The shaded regions depict beams generated by $150-210$-day zonal wind at $77^{\circ}$ E: Kelvin wave (blue), incident Rossby wave (red), and reflected Rossby wave (green). The thick solid lines show the corresponding 180-day ray path. (b) The region occupied by all the ray paths of a 180-day wave when the wind blows along the entire equatorial Indian Ocean. The incident (reflected) Rossby wave occupies the blue (green) shaded region. Both Kelvin wave and incident (reflected) Rossby wave occupy the purple (blue) region. The solid lines show the ray paths generated at 

${ }_{525}$ The dark brown region is the bathymetry and the hatched region is the shadow the ray paths act as the lower limit below which the wave cannot be detected. The grey shaded region is the shadow zone where no ray paths reach. (c) Ray paths of reflected Rossby waves for different meridional modes generated by a 180day Kelvin wave from the western boundary. Higher modes have steeper slopes and smaller shadow zones. (d) Ray paths of reflected Rossby waves generated by Kelvin waves with different frequencies. The area of the shadow zone decreases with increasing frequency. (e) Ray paths of 180-day Kelvin waves and reflected Rossby waves with different $N_{b}^{2}$ profiles to show the effect of stratification on the shadow zone. The differences are negligible between the summer (red) and the winter (blue) profiles. (f) The 90-day Kelvin wave ray paths from the western boundary of the Indian Ocean and the maritime continent. The reflected Rossby wave is evident only in the Indian Ocean. In the Pacific Ocean, the Kelvin wave reaches $4000 \mathrm{~m}$ without generating reflected Rossby waves because of the larger basin size. The arrowheads in all panels indicate the direction of the ray paths, and the ray paths are shown only for the first meridional mode except in panel (c).

Fig 4 The panels show ray paths for Kelvin and Rossby waves for four different periods: a) 180 day, b) 120 day, c) 90 day, and d) 30 day. The shaded color represent the log of the normalized periodogram of the zonal currents. Blue solid (thin) line is the Kelvin ray path generated from the western boundary $\left(59.5^{\circ} \mathrm{E}\right)$. The orange and red solid lines are Rossby beams generated at the eastern boundary for mode 1 and 5, respectively. The green and purple dashed lines are the Rossby beams generated after the Kelvin wave (blue solid) reflects from the eastern boundary.

the boundaries. As waves cannot be generated by winds beyond these boundaries, 
526 zone for the mode 5 reflected Rossby waves. The white vertical line/circles show data availability. The lines represent ADCP data and the circles represent data from current meters. The ray paths are calculated based on the WKB theory. The colour levels for the periodogram are not the same for all panels.

Fig. 5 Figure showing periodogram of winds at the equator. The power is plotted in log scale after detrending and applying a Hamming window. The ordinate is periods and the abscissa is the longitude. The wind data used to calculate the periodogram extends from 2000-2016. 

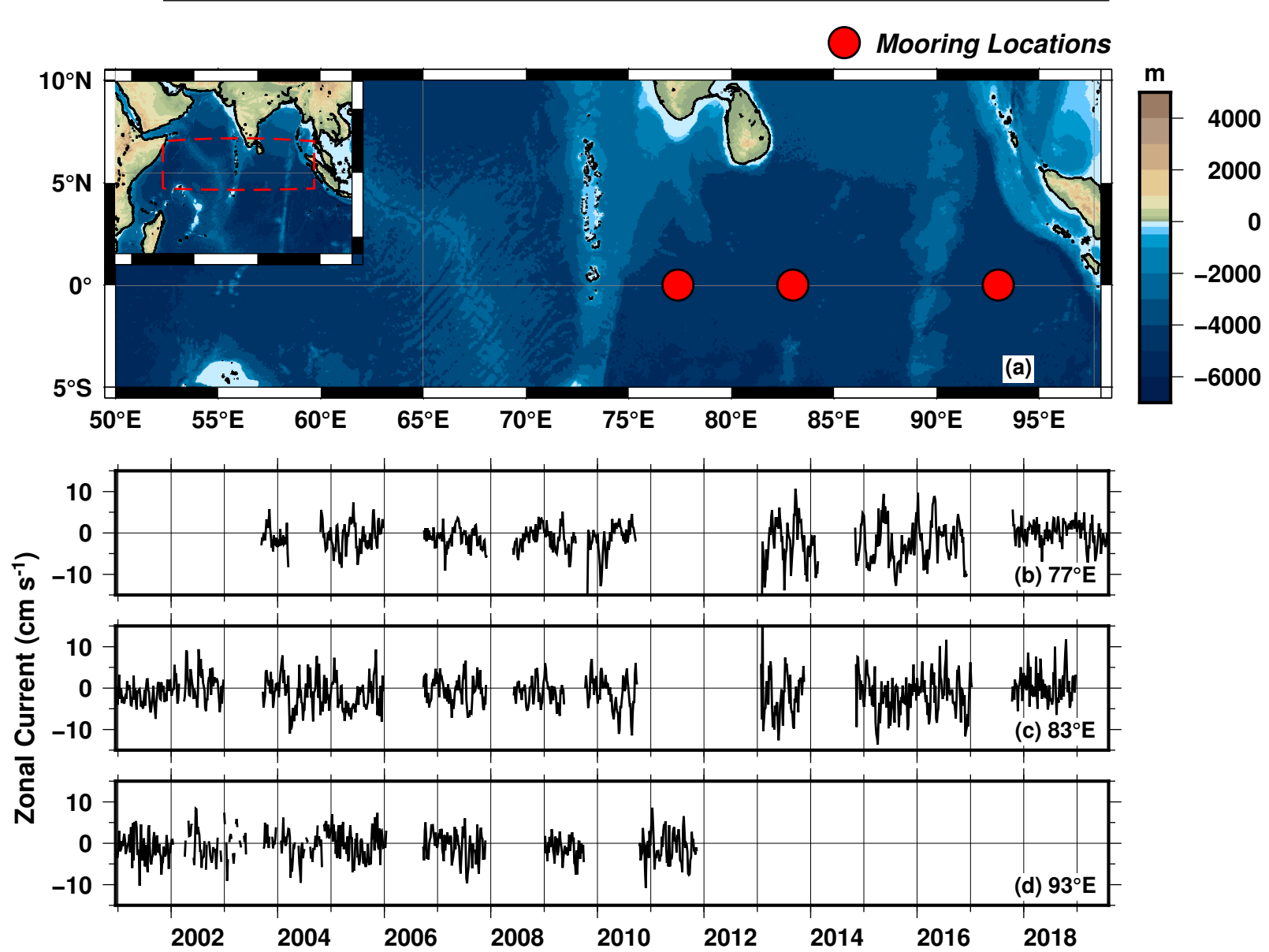

Fig. 1 (a) Map showing the mooring locations. The background is the bathymetry from Sindhu et al. (2007). The inset shows map of north Indian Ocean. The red dashed line is the region of interest. Zonal currents at $4000 \mathrm{~m}$ at (b) $77^{\circ} \mathrm{E}$, (c) $83^{\circ} \mathrm{E}$, and (d) $93^{\circ} \mathrm{E}$. 

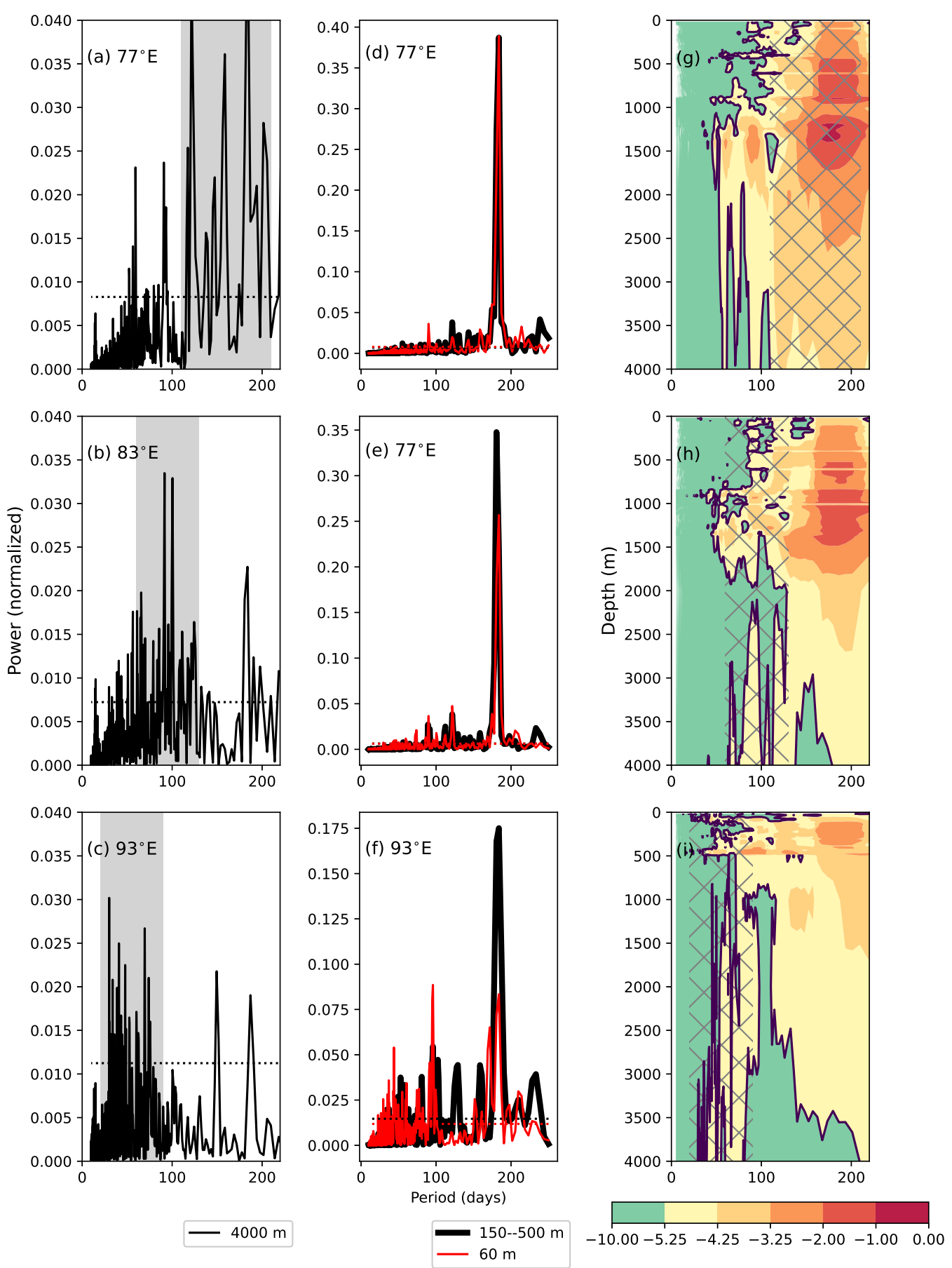

Fig. 2 Normalized Lomb periodogram of zonal currents at $4000 \mathrm{~m}$ depth: (a) $77^{\circ} \mathrm{E}$, (b) $83^{\circ} \mathrm{E}$, and (c) $93^{\circ} \mathrm{E}$. The dashed horizontal line shows the $1 \%$ false alarm probability. The gray shaded region highlights the shift in dominant frequencies from west to east. (d)-(f) Same as (a)-(c) but for currents at $60 \mathrm{~m}$ (black) and 150-500 m (red). The spectra for the 150-500 m current is computed after averaging the current over this depth range. Note that the ordinate does not have the same limits. (g)-(i) Normalized Lomb periodogram of zonal currents with depth. As the difference in power is large between the surface and bottom currents, the periodogram is smoothed and plotted in log scale to highlight the shift in dominant frequency with depth. The hatched area is equivalent to the gray shaded region in panels (a)-(c). 

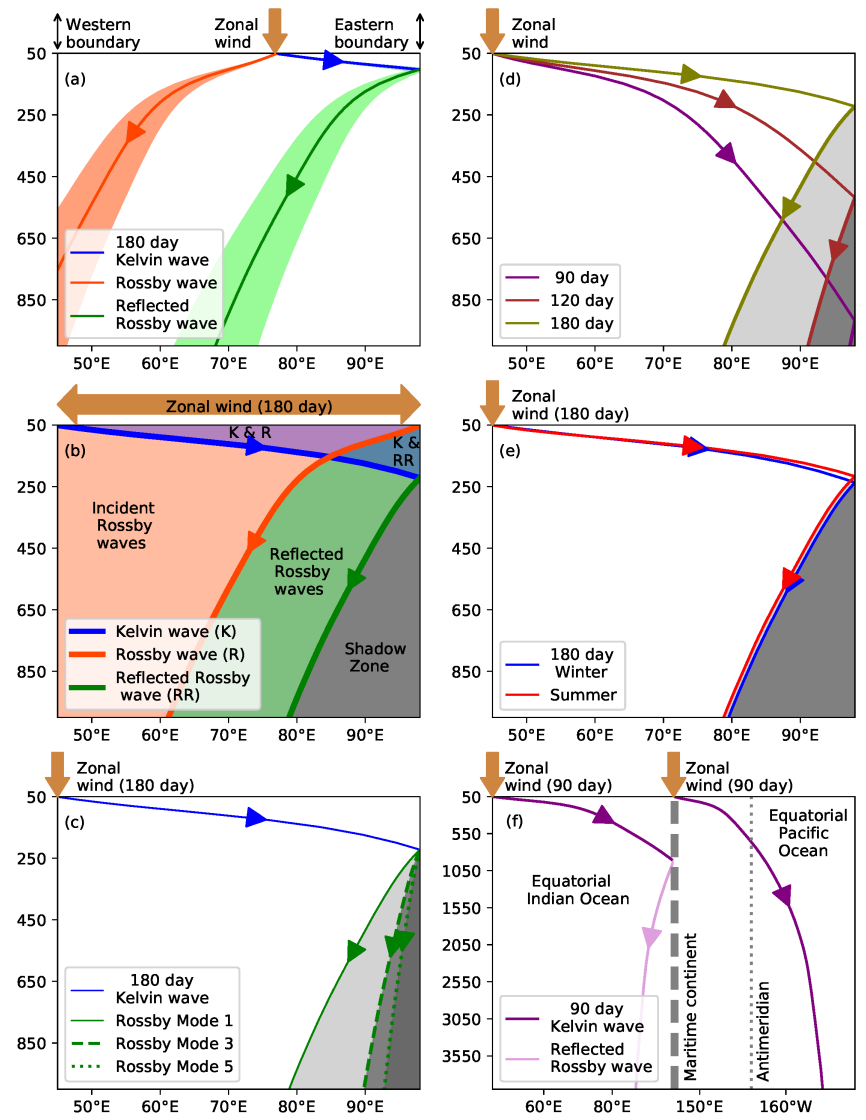

Fig. 3 Schematic of the ray paths in the equatorial Indian Ocean. (a) The shaded regions depict beams generated by 150-210-day zonal wind at $77^{\circ} \mathrm{E}$ : Kelvin wave (blue), incident Rossby wave (red), and reflected Rossby wave (green). The thick solid lines show the corresponding 180-day ray path. (b) The region occupied by all the ray paths of a 180-day wave when the wind blows along the entire equatorial Indian Ocean. The incident (reflected) Rossby wave occupies the blue (green) shaded region. Both Kelvin wave and incident (reflected) Rossby wave occupy the purple (blue) region. The solid lines show the ray paths generated at the boundaries. As waves cannot be generated by winds beyond these boundaries, the ray paths act as the lower limit below which the wave cannot be detected. The grey shaded region is the shadow zone where no ray paths reach. (c) Ray paths of reflected Rossby waves for different meridional modes generated by a 180-day Kelvin wave from the western boundary. Higher modes have steeper slopes and smaller shadow zones. (d) Ray paths of reflected Rossby waves generated by Kelvin waves with different frequencies. The area of the shadow zone decreases with increasing frequency. (e) Ray paths of 180-day Kelvin waves and reflected Rossby waves with different $N_{b}^{2}$ profiles to show the effect of stratification on the shadow zone. The differences are negligible between the summer (red) and the winter (blue) profiles. (f) The 90-day Kelvin wave ray paths from the western boundary of the Indian Ocean and the maritime continent. The reflected Rossby wave is evident only in the Indian Ocean. In the Pacific Ocean, the Kelvin wave reaches $4000 \mathrm{~m}$ without generating reflected Rossby waves because of the larger basin size. The arrowheads in all panels indicate the direction of the ray paths, and the ray paths are shown only for the first meridional mode except in panel (c). 


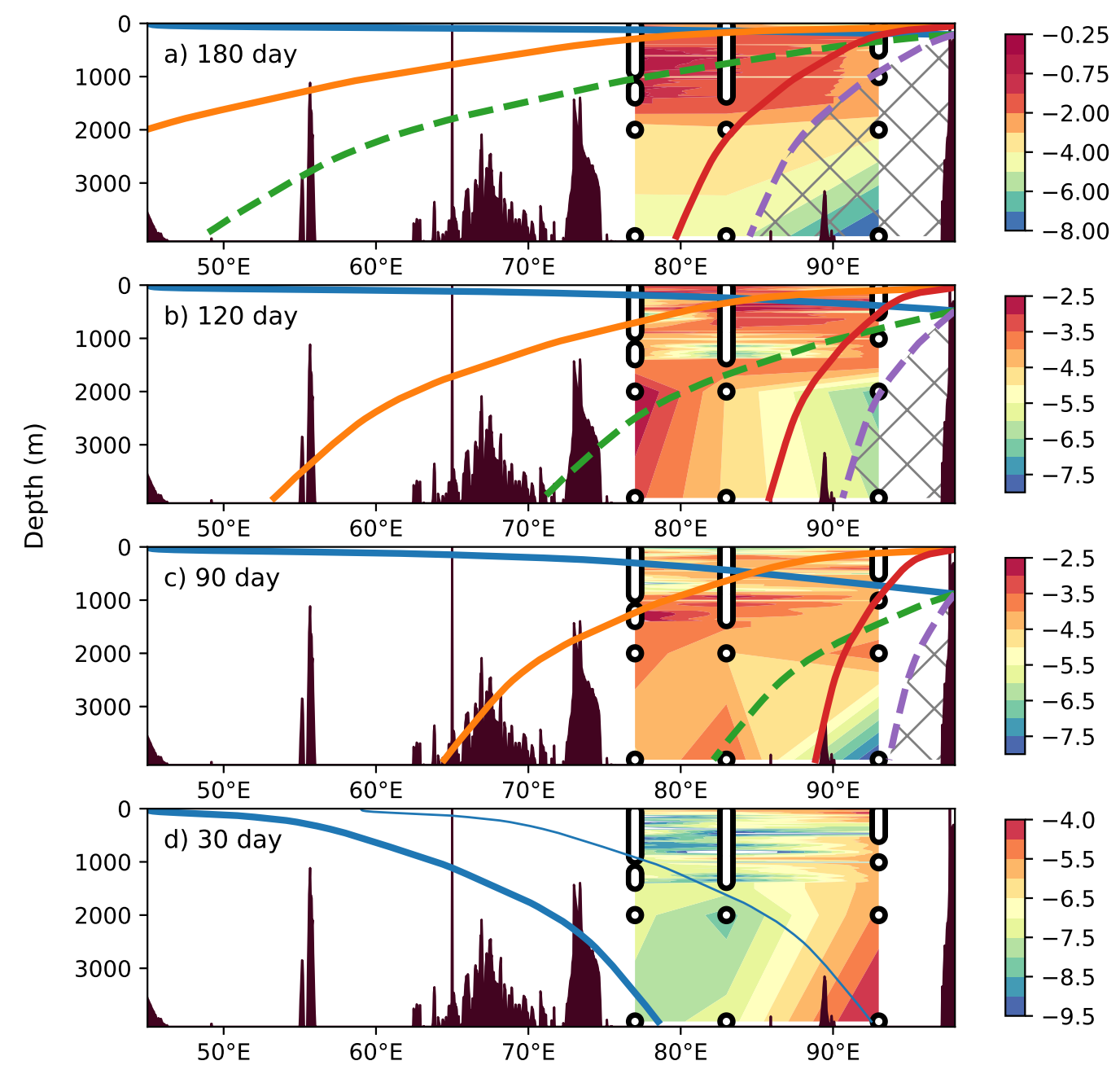

Kelvin Rossby Mode $1=$ Rossby Mode 1

Rossby Mode 5

Rossby Mode 5

Fig. 4 The panels show ray paths for Kelvin and Rossby waves for four different periods: a) 180 day, b) 120 day, c) 90 day, and d) 30 day. The shaded color represent the log of the normalized periodogram of the zonal currents. Blue solid (thin) line is the Kelvin ray path generated from the western boundary $\left(59.5^{\circ} \mathrm{E}\right)$. The orange and red solid lines are Rossby beams generated at the eastern boundary for mode 1 and 5 , respectively. The green and purple dashed lines are the Rossby beams generated after the Kelvin wave (blue solid) reflects from the eastern boundary. The dark brown region is the bathymetry and the hatched region is the shadow zone for the mode 5 reflected Rossby waves. The white vertical line/circles show data availability. The lines represent ADCP data and the circles represent data from current meters. The ray paths are calculated based on the WKB theory. The colour levels for the periodogram are not the same for all panels. 


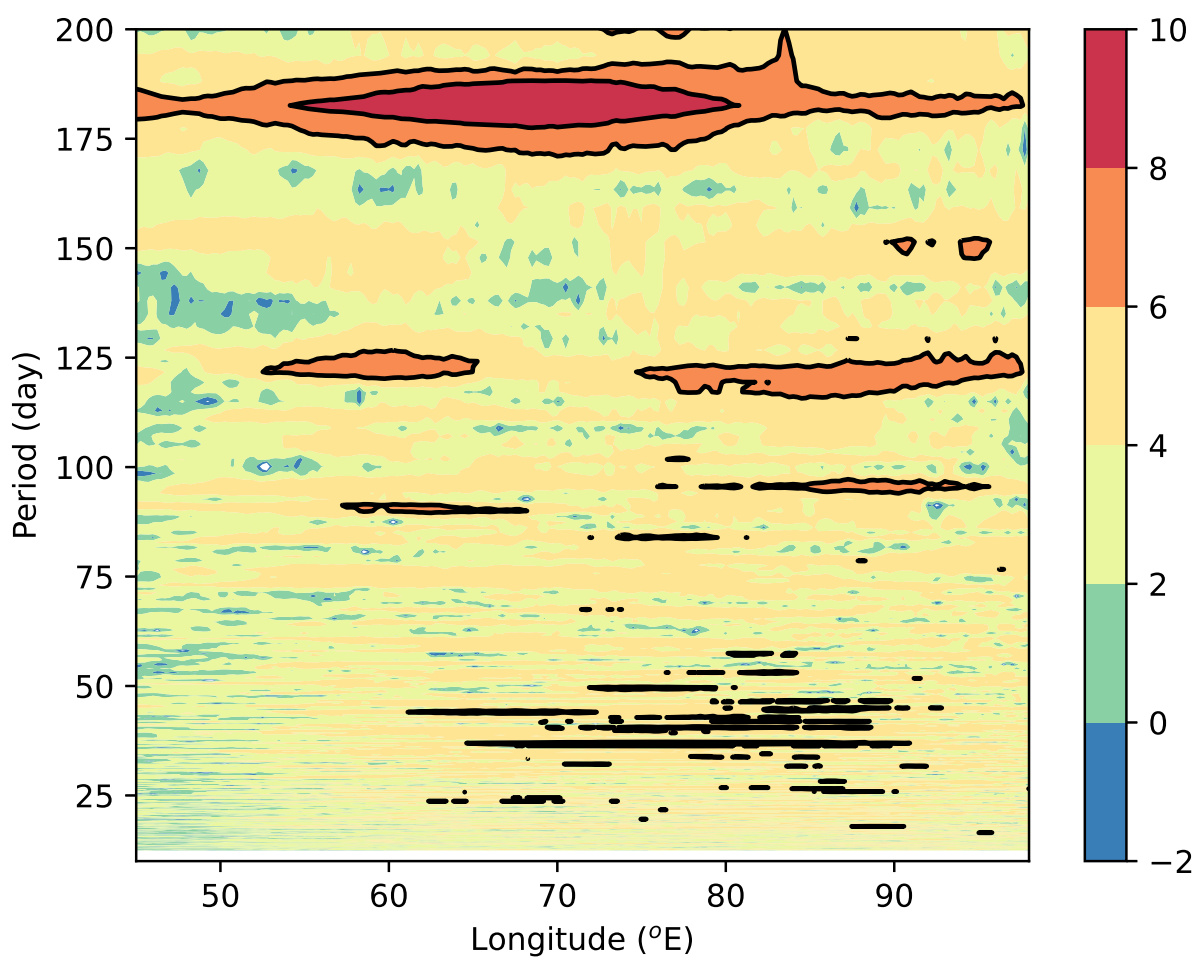

Fig. 5 Figure showing periodogram of winds at the equator. The power is plotted in log scale after detrending and applying a Hamming window. The ordinate is periods and the abscissa is the longitude. The wind data used to calculate the periodogram extends from 2000-2016. 